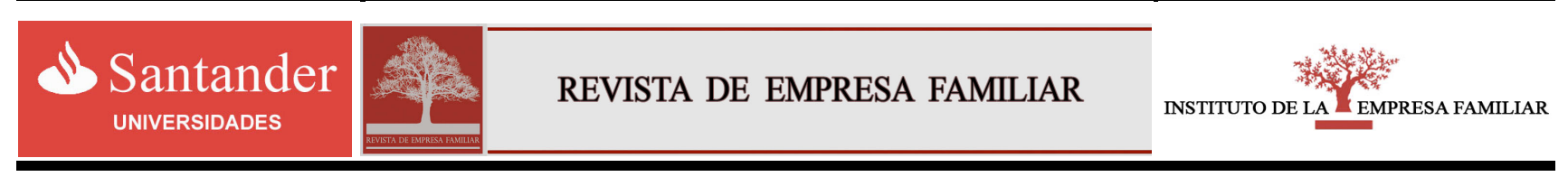

www.revistadeempresafamiliar.uma.es

\title{
La mujer en la empresa familiar
}

\section{Women in the family business}

\author{
Maribel Rodríguez Zapatero ${ }^{\mathrm{a}, *} \cdot$ Magdalena Rodriguez Jiménez ${ }^{\mathrm{b}}$ \\ ${ }^{\mathrm{a}, \mathrm{b}}$ Departamento de Estadística, Econometría, Investigación Operativa y Organización de Empresas. \\ Universidad de Córdoba,14005 (Spain)
}

\begin{tabular}{l} 
D A T O S A R T Í C U L O \\
\hline Historial: \\
Recibido 11-07-2011 \\
Aceptado 09-11-2011 \\
Palabras clave: \\
Empresa familiar \\
Mujer \\
Conciliación \\
conflicto \\
Códigos JEL: \\
M1
\end{tabular}

\section{A R T I C L E I N F O \\ Article history: \\ Received 11 July 2011 \\ Accepted X November 2011}

Keywords:

Family business

Women

Conciliation

Conflict

JEL codes:

$\mathrm{M} 1$

\begin{abstract}
RES UMEN
El objetivo perseguido por el presente trabajo de investigación es llegar a un mejor entendimiento del papel que juega la mujer en la empresa familiar. Se utilizó un cuestionario contestado por ciento diecinueve mujeres, que recabó junto a información demoscópica datos relativos a quién tomó la decisión de entrar a trabajar en la empresa familiar, ventajas y desventajas de trabajar en la empresa familiar, recomendaciones a las hijas para su unión al negocio familiar y tipos de relaciones y conflictos en la empresa familiar. Pese a que la limitación de este estudio reside en el tamaño muestral creemos que la investigación deja una puerta abierta a futuros estudios.
\end{abstract}

\begin{abstract}
A B S T R A C T
This research paper aims a better understanding of women's roles within family business. One hundred and nineteen women answered a questionnaire designed to obtain demoscopic information and questions like who decided to join the family business, advantages and disadvantages of working in family firm, recommendations to daughters and types of relationship and conflict perceived. Our relatively small sample size is a study limitation to be considered, although we strongly think this research open a door for future research.
\end{abstract}

* Autor de contacto. Tel.: +34957218747

Correoselectrónicos: es3rozai@uco.es,pu2rojim@uco.es 


\section{Conciliación y conflicto en la mujer empresaria familiar}

Conseguir encontrar un cierto equilibrio entre familia y trabajo es un proceso muy complejo para aquellas mujeres que trabajan fuera del hogar y además desarrollan sus funciones de esposas y madres.

En el caso de las mujeres que trabajan en empresas de la familia ese equilibrio es aún más complejo si se compara con hermanos que no trabajan en la empresa de la familia. Todavía el equilibrio es más complejo si la mujer trabaja para la empresa familiar $y$ no percibe remuneración alguna por razones de legislación o culturales.

Creemos que se precisa comprender a la mujer casada que desarrolla un trabajo en la empresa de su familia, así como el contexto en el que se desarrolla su tarea, los procesos que condicionan la ejecución de su trabajo y cómo todo ello influye en su bienestar personal. Stoner, C.R. (1990), así como otros trabajos, exploraron el conflicto en que viven las mujeres que trabajan en su propia empresa y además dirigen y administran el hogar, cuando se trata de pequeñas y medianas empresas.

Poder conciliar las responsabilidades que demandan el hogar y el trabajo es un problema que cada día experimentan con mayor intensidad empresarios y empleados. $\mathrm{La}$ incorporación de la mujer al trabajo no ha dado lugar a cambios en roles de la mujer en el hogar, pues están por definir los papeles a representar en el hogar por el hombre y la mujer.

Esa inercia quizás puede deberse al alto valor que la sociedad da a la maternidad en relación con la paternidad. La mujer que trabaja siente más angustia a la hora de conciliar su carrera profesional, el cuidado de la prole y la administración del hogar. Esa conciliación puede ser más difícil aún para aquella mujer que trabaja no para otros sino para su propia empresa, pues las fronteras entre trabajo y familia son difusas y además la mujer desarrolla con frecuencia un trabajo sin remuneración y/o sin la debida titulación profesional.

El conflicto entre familia y negocio familiar es más intenso en la mujer que en el hombre, pues la mujer se orienta a construir y mantener relaciones entre los familiares y de éstoson la empresa familiar. Se reconoce generalmente que en la mujer recae la responsabilidad de contribuir a los objetivos familiares de crecimiento y continuidad del negocio a través de su función pacificadora de la familia. Sobre estas funciones de la mujer en la familia empresaria destacan los trabajos de Danes y McTavish (1997); Danes, Haberman y McTavish (2005); Danes y Olson (2003) o Salganicoff (1990). Se viene conjeturando que el autoempleo se percibe como una opción personal que conduce a equilibrar las responsabilidades inherentes al trabajo y la familia tal y como indican Kean, Memeyer y Maupin (1994). No obstante, las personas autoempleadas generan un mayor nivel de conflicto entre familia y trabajo y una menor satisfacción si se compara con trabajadores por cuenta ajena. En el caso de la mujer autoempleada la presión del tiempo, el tamaño de la familia, el apoyo que reciba, la bondad de la relación marital y la satisfacción en su trabajo influyen de manera notable en la conciliación entre el trabajo y la familia y en el conflicto que esta dualidad genera. El conflicto entre funciones (familia-trabajo) debe ser manejado de modo que se consiga la estabilidad del sistema completo (familia y empresa), pues la intersección familia-empresa requiere gran cantidad de negociación y gestión.

La acumulación de tareas produce infelicidad y ansiedad y reduce la sensación de bienestar. Ya la teoría de la gestión de la familia postula que las mujeres incorporan sus evaluaciones subjetivas a las discrepancias existentes entre el nivel deseado de logro de los objetivos familiares y el nivel propio de felicidad (Olson y Danes, 2003). La felicidad para la mujer que trabaja en su empresa familiar es una combinación compleja de consumo, familia, red de apoyo, condiciones de trabajo, libertad y posesión de bienes que deben mantener una cierta armonía entre lo que se quiere y/o que se tiene. Es obvio que la relación entre trabajo, gestión de la empresa y de la familia para un mujer casada que trabaje en la empresa familiar es algo crítico y necesario para controlar y crear una energía positiva que ayude a la empresa, sin 
drenar demasiados recursos que se deben dedicar a la familia. Ese ir y venir de la familia a la empresa y viceversa afecta al bienestar de la mujer, madre y esposa. Para un análisis modélico podemos consultar el trabajo de Stafford (1999).

La intensidad con la que la mujer casada juega estos papeles depende de su edad, como indicador de las cambiantes necesidades que la mujer siente de integración social. También depende de su nivel de educación y formación, pues ello afecta a su capacidad de gestión en la empresa familiar y al compromiso con que asume ciertas responsabilidades. Desde luego que su estado de salud permitirá o no que la mujer pueda dedicar energías a esa doble tarea; así como el número de hijos menores de edad. La competición por tiempo y energías que demandan tanto la empresa como la familia, en situación de escasez de flujo de caja del negocio, afecta a la marcha tanto de la empresa como de la familia y de sus componentes. Esta aseveración es mostrada por varios trabajos, entre ellos, Zuiker, V.S., Lee, Y.G., Olson, P.D., Danes, S.M., Danes, A.N., Vanguilder-Dik \& Katras, M.J. (2002).

\section{Esquema conceptual}

Para analizar el papel de la mujer y su nivel de satisfacción en la empresa familiar hemos seguido el modelo de Empresa Familiar sostenible que esbozaron Stafford, K., Duncan, Danes y Winter (1999).

El modelo conceptual en el que nos basamos para situar a la mujer en el marco de la empresa familiar es aquel que reconoce no sólo que tanto la familia como la empresa son entidades sociales viables, sino también que existe un territorio de confluencia e intersección entre los dos sistemas anteriores. Cada sistema necesita recursos para sostenerse, expresados en tiempo, energía, dinero, expectativas, responsabilidades y objetivos a conseguir, así como transacciones entre individuos constituyentes de los mismos a fin demandar aquellos recursos, hacer funcionar los sistemas y resolver sus contradicciones. Es obvio que cada sistema, familia y empresa, necesitan recursos específicos y también es obvio que se producen drenajes de recursos de un sistema a otro en las dos direcciones.

Dentro de la familia y de la empresa cada familiar fija sus estándares para valorar recursos y valorar logros. Cuando el logro no se materializa, según cada autofijación o no se asignan los recursos necesarios, se produce una dinámica de acción, y a veces, una discrepancia y un conflicto. En este juego de asignación de recursos, percepción de logros y discrepancias, la mujer casada en el seno de la familia empresaria es un actor más del juego. La perturbación surge en el proceso de transferencias de recursos de la familia a la empresa y viceversa, cuya valoración es subjetiva por parte de los actores.

La mujer y el hombre tienen dimensiones objetivas y subjetivas de lo que está sucediendo en la familia y en su empresa. Esas dimensiones no son intercambiables; ni lo son sus conceptualizaciones ni sus significados.

Las mediciones objetivas son fácilmente asimilables pero no lo son las mediciones subjetivas del éxito o fracaso, del esfuerzo o del compromiso. La dedicación de tiempo y energías al cuidado de la prole y a la gestión del negocio produce discrepancias en el seno de la familia nuclear empresaria y son fuente de tensión y conflicto entre esposos. A menudo la sostenibilidad del sistema completo obliga a programar los procesos de la familia y de la empresa de modo conjunto. $\mathrm{Si}$ esta coprogramación no se verifica surgen dificultades, tensiones y discrepancias que pueden hacer que la empresa y la familia no sean sostenibles.

Con la finalidad de investigar las interrelaciones entre familia y empresa familiar en relación con las percepciones que de ello tienen sus constituyentes, la presente investigación analiza las percepciones de la mujer en la empresa familiar.

\section{Metodología del estudio}

Se ha configurado un cuestionario específico para mujeres con el fin de indagar y profundizar en el conocimiento del género femenino y así 
entender mejor sus comportamientos en relación con empresa familiar.

Elaboramos un cuestionario con cinco constructos claramente diferenciados. Con el primero de ellos obtenemos datos demoscópicos tales como edad, estado civil, estado laboral, nivel de formación, etc. En el segundo constructo incluimos 3 bloques de preguntas.

En una primera fase se preguntó a las mujeres quién tomó la decisión de que ella entrara a trabajar en la empresa familiar así como que eligieran entre una serie de propuestas cuáles eran las ventajas y desventajas de trabajar en una empresa familiar.

En tercer lugar preguntamos a las mujeres sobre las recomendaciones que harían a sus hijas en caso que éstas entraran a trabajar en la empresa familiar, lo que nos proporciona información relevante sobre su propia experiencia en la empresa familiar. En cuarto lugar recopilamos información sobre las relaciones con sus progenitores y hermanos, en su caso, y sobre el posicionamiento sobre la relación familia-trabajo y el relevo generacional.

La muestra está constituida por 119 mujeres. Sobre la población total graduada, 384 alumnos, las mujeres pesan un $37 \%$ (144 mujeres). Nuestra muestra de 119 mujeres representa el $82,6 \%$ del total mujeres graduadas. El cuestionario se les facilitó a las mujeres asistentes a los seminarios de formación sobre Empresa Familiar que imparte la Universidad de Córdoba anualmente.

\section{Resultados del estudio}

\subsection{Edad}

La distribución de edad de nuestra muestra de estudio se recoge en la tabla 1.

El $81,51 \%$ de las mujeres estudiadas tienen entre 20 y 50 años. El estrato de mayor representación es el intervalo 30-39 con un peso de $32,77 \%$. Es interesante conocer esta distribución porque partimos de la hipótesis que la variable edad influye en las contestaciones de las mujeres.

\section{Tabla 1}

Distribución de la muestra por edad.

\begin{tabular}{ccc}
\hline Edad & $\mathrm{N}^{\mathrm{o}}$ mujeres & $\%$ \\
\hline $20-29$ & 35 & $29,41 \%$ \\
$30-39$ & 39 & $32,77 \%$ \\
$40-49$ & 23 & $19,33 \%$ \\
$50-59$ & 15 & $12,61 \%$ \\
$60-70$ & 7 & $5,88 \%$ \\
\hline Total general & 119 & $100,00 \%$ \\
\hline
\end{tabular}

Fuente: elaboración propia

\subsection{Nivel educativo de la muestra}

La formación o nivel educativo de una persona influye, al igual que la variable edad, en las contestaciones posibles derivadas del cuestionario. Nuestra muestra podría definirse como una muestra de mujeres con alto nivel de formación a la luz de los resultados obtenidos en el análisis de datos, no obstante, no hay que olvidar que nuestra muestra es dirigida por tratarse de alumnos que cursan los seminarios de formación sobre Empresa Familiar de la Universidad de Córdoba. La tabla 2 informa de los resultados obtenidos sobre el nivel formativo.

\section{Tabla 2}

Distribución de la muestra por nivel formativo.

\begin{tabular}{lll}
\hline $\begin{array}{c}\text { Nivel de } \\
\text { formación }\end{array}$ & $\mathrm{N}^{\mathrm{o}}$ mujeres & $\mathbf{\%}$ \\
\hline Bachiller & 15 & $12,61 \%$ \\
Elemental & 20 & $16,81 \%$ \\
Formación & 19 & $15,97 \%$ \\
Profesional & 5 & $4,20 \%$ \\
Otro & 60 & $50,42 \%$ \\
Universidad & 119 & $100,00 \%$ \\
\hline Total &
\end{tabular}

El gráfico 2 clasifica la muestra en intervalos de edad y grado de formación por intervalo.

\subsection{La mujer como trabajadora activa en la empresa familiar}

No todas las mujeres encuestadas trabajan de manera activa en la empresa familiar; tan solo 


\section{Gráfico 1}

Distribución de la muestra por edad y nivel formativo.

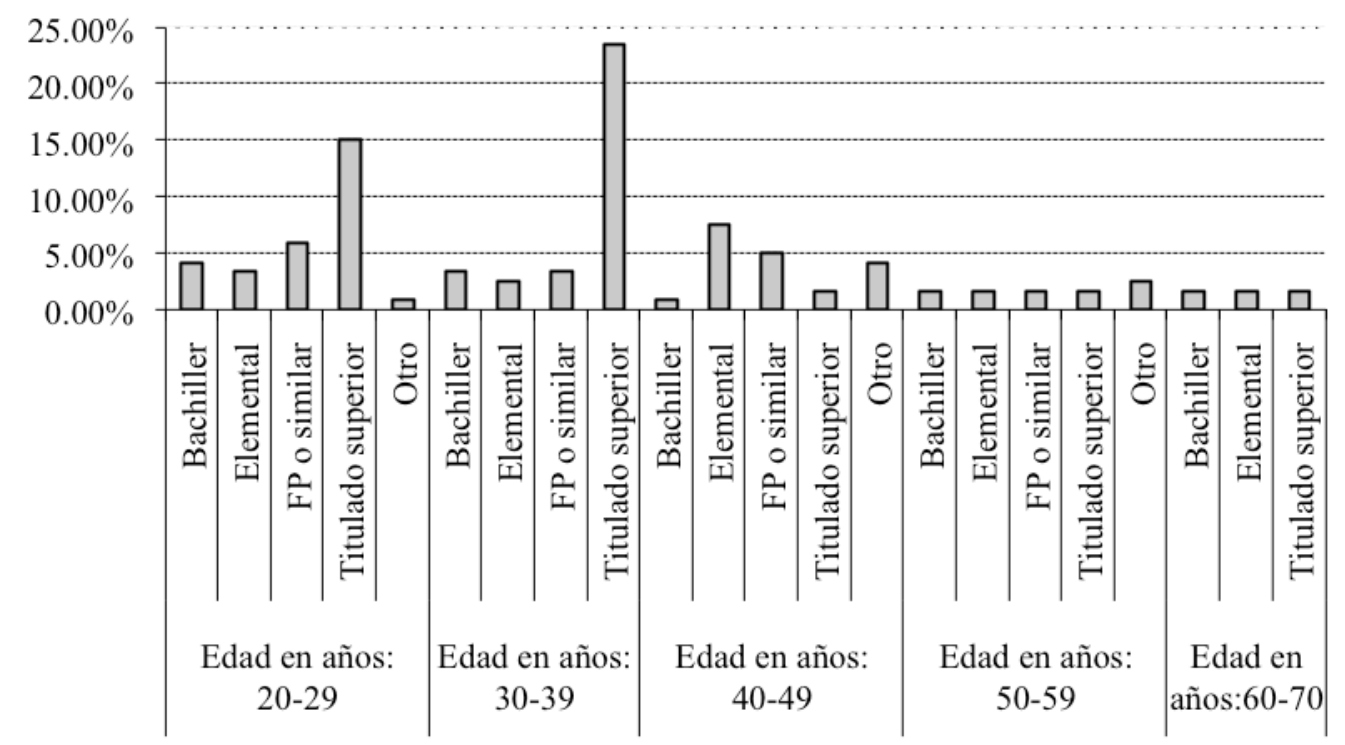

Fuente: elaboración propia

un $71,43 \%$ trabaja frente a un $28,57 \%$ que no lo hace. Nos interesa saber de este $71,43 \%$ de mujeres, qué edad tenían cuando ocuparon un puesto de dirección en la empresa y qué edad tenían cuando ocuparon la gerencia.

La edad de comienzo a trabajar en la empresa familiar se mueve desde los 20 años hasta los 55 años (véase tabla 3).

\section{Tabla 3}

Edad de comienzo a trabajar en la empresa familiar.

\begin{tabular}{lcccccc}
\hline $\begin{array}{l}\text { Edad de } \\
\text { entrada }\end{array}$ & \multicolumn{7}{c}{ Edad actual } \\
a & $20-$ & $30-$ & $40-$ & $50-$ & $60-$ & \\
trabajar & 29 & 39 & 49 & 59 & 70 & Total \\
\hline $10-19$ & 6 & 4 & 8 & 1 & & 19 \\
$20-29$ & 19 & 24 & 8 & 2 & & 53 \\
$30-39$ & & 5 & 1 & 2 & 2 & 10 \\
$40-49$ & & & 1 & 1 & & 2 \\
$50-59$ & & & & & 1 & 1 \\
\hline Total & 25 & 33 & 18 & 6 & 3 & 85 \\
\hline$\%$ & 29,4 & 38,8 & 21,2 & 7,1 & 3,5 & 100 \\
\hline
\end{tabular}

Fuente: elaboración propia

La orquilla es muy amplia y responde a diferentes motivaciones que serán analizadas más adelante en nuestra investigación. No obstante la edad de incorporación de la mujer a la empresa familiar se distribuye con una media de 24 años y una desviación estándar cercana a 6,88 , lo que nos explica cómo factores externos provocan la entrada de la mujer (muerte repentina del fundador, cola derecha de la distribución, necesidad de mano de obra (cola izquierda de la distribución). Cuando el puesto de dirección se analiza, las mujeres en activo en la empresa familiar ocuparon puestos de dirección tan sólo en un 38,82\% de los casos.

La edad media con que la mujer en la empresa familiar asume un cargo de responsabilidad se distribuye con una media de 31 años y una desviación estándar de 7,14. Representan el $38.82 \%$ de la muestra (véase tabla 4 y gráfico 2)

Podemos observar cómo el porcentaje de mujeres en activo con puestos de responsabilidad $(38,82 \%)$ es inferior al porcentaje de mujeres que trabajan en la empresa familiar (71,43\%). Si además analizamos si alguna de las mujeres ocupa la gerencia, se puede concluir que la responsabilidad es inversamente proporcional al 
número de mujeres activas en la empresa familiar.

\section{Tabla 4}

Porcentaje de mujeres que ocupan cargos de responsabilidad en la empresa familiar.

\begin{tabular}{lc}
\hline Nivel de responsabilidad & Frecuencia (\%) \\
\hline Puestos de Responsabilidad & $38,82 \%$ \\
Sin responsabilidad & $61,18 \%$ \\
Total & $100,00 \%$ \\
\hline
\end{tabular}

Fuente: elaboración propia

4.4. La decisión de entrar a trabajar en la empresa familiar. Ventajas y desventajas del trabajo en la empresa familiar. Recomendaciones a la futura hija

Analizaremos la decisión de entrar a trabajar o no en la empresa familiar, las respuestas figuran en la tabla 5 .

\section{Tabla 5}

Decisión de entrar a trabajar en la empresa familiar.

\begin{tabular}{lll}
\hline Decidió usted trabajar en EF & Frecuencia & $(\%)$ \\
\hline No & 41 & 34,45 \\
Sí & 78 & 65,55 \\
\hline Total & 119 & 100 \\
\hline
\end{tabular}

Fuente: elaboración propia
Un $65,55 \%$ de las mujeres decidió entrar a trabar en la empresa familiar y un $34,45 \%$ decidió no hacerlo por propia voluntad.

Las ventajas y desventajas que encontraban las mujeres encuestadas en el desempeño del trabajo en la empresa familiar se analizan a continuación. Sus respuestas pusieron de manifiesto que la mujer se mueve por el compromiso, por la lealtad y por los valores de la familia y que otras cuestiones tales como la libertad o flexibilidad horaria no son ventajas claves pese a lo que en un principio pudiera pensarse.

\subsection{Ventajas y desventajas de trabajar en la empresa familiar}

Entre 8 ventajas y 6 desventajas se pidió a las mujeres que elaboraran un ranking de tres ventajas y desventajas percibidas por ellas derivadas del trabajo en la empresa familiar. El análisis de las ventajas se plasma a continuación.

Las ocho ventajas expuestas a las encuestadas para elaborar un ranking de tres fueron las siguientes:

V1-Buena reputación del fundador lo que te ayuda a abrirte camino

\section{Gráfico 2}

Distribución de la edad de ocupación de puestos de responsabilidad.

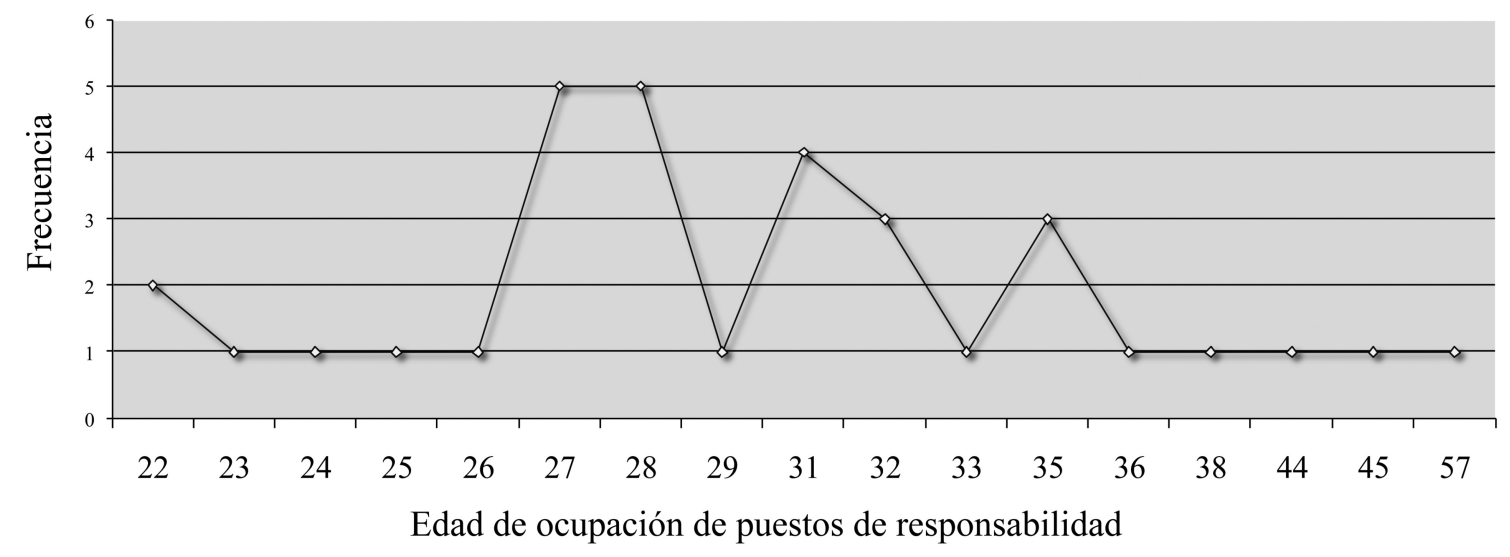

Fuente: elaboración propia 
V2-Orgullo de continuar con la tradición familiar

\section{V3-Flexibilidad horaria}

V4-Confortabilidad en el lugar de trabajo

V5-El compromiso de todos los familiares en el negocio

V6-La lealtad entre familiares

V7-Poder dar tiempo a la vida familiar.

V8-Compartir la misma escala de valores

El mapa de ventajas que resultó del análisis de las encuestas se recoge a continuación.

El orgullo de continuar la tradición familiar se sitúa como la primera de las ventajas escogidas por la muestra de mujeres estudiadas (V2).

Le siguen en orden de importancia el compromiso de todos los familiares con el negocio (V5) y compartir la misma escala de valores (V8).

Por el lado de las desventajas, los pronunciamientos obtenidos se reflejan a continuación, de las seis posibilidades ofrecidas:

D1-No tener tiempo para la vida familiar

D2-La constante comparación con el modo de hacer del fundador o del jefe familiar

D3-Trabajar demasiadas horas

D4-La responsabilidad de llevar la empresa para financiar la jubilación de mis padres o de mis tíos

D5-El conflicto familiar en el trabajo se traslada a la familia

D6-No tener vida privada fuera de la familiar.

En orden de importancia D5, D1, D3 se configuran como las principales desventajas percibidas por las mujeres por el hecho de trabajar en la empresa familiar.

El conflicto familiar en el trabajo se traslada a la familia, aparece como la desventaja principal, seguida de no tener tiempo para la vida familiar al trabajar demasiadas horas.

\subsection{Recomendaciones a sus hijas}

Preguntamos de entre 10 posibles recomendaciones que le harían a su hija si decidiesen entrar a trabajar en la empresa familia, cuáles señalarían prioritariamente.

Las 10 posibles recomendaciones se reflejan a continuación:

R1-Terminar la formación y definir los objetivos profesionales antes de entrar a trabajar o de tomar el mando de la empresa

R2-Encontrar alguien que le ayude a aprender la dirección

R3-Tomar experiencia en otra organización no familiar para entender el entorno y la cultura empresarial

R4-Aprender finanzas y financiación de la empresa

R5-No tener miedo de emplear a gente que sepan más que ella

R6-No asumir la gerencia si no se tiene una verdadera pasión por dirigir

R7-Debe demostrar a otros que está dispuesta a trabajar duramente desde el primer día

R8-Competir con el hombre, pero saberse respetar como mujer; no ser agresiva pero sí ser firme

R9-Confiar en su propio instinto

R10-Fijar sus estándares y no necesariamente lo de sus antecesores en la empresa

$\mathrm{R} 1$, R3 y R5 serían las recomendaciones que las madres darían a sus hijas.

La formación y experiencia previa a la entrada a la empresa familiar es considerada por la madre como paso clave para ganar respeto en los trabajadores no familiares de la empresa familiar.

La formación y la experiencia previa en otras empresas puede permitir y ayudar a la mujer en el futuro a asumir responsabilidades y gobernar equipos humanos sin titubeos. 
4.7. Las relaciones de la mujer con el gerente y con su madre y sus hermanos

Intentamos descubrir cómo son las relaciones de la muestra de mujeres encuestadas con el gerente familiar o no, con su madre, si no trabaja en la empresa familiar, y con sus hermanos en el caso de que los hubiese.

Para valorar las relaciones con la gerencia de la empresa se dieron a escoger tres posibilidades:

-No hubo ningún conflicto entre ambos $(51,56 \%)$

-Me trataba como a una hija en lugar de cómo a una empleada $(26,56 \%)$

-Me trataba más duramente que a cualquier otro empleado (21,88\%)

La figura con mejor puntuación en relaciones sin conflicto es la relación mantenida con el gerente de la empresa familiar, en un porcentaje del 51,56\%; Este porcentaje se eleva cuando las mujeres respondieron a las relaciones mantenidas con su madre y con sus hermanos, tal y como refleja el gráfico 4.

Como se observa en el gráfico 4, la relación maternal no ha planteado conflicto alguno en el

\section{Gráfico 3}

Relaciones sin conflicto de la mujer en la empresa familiar.

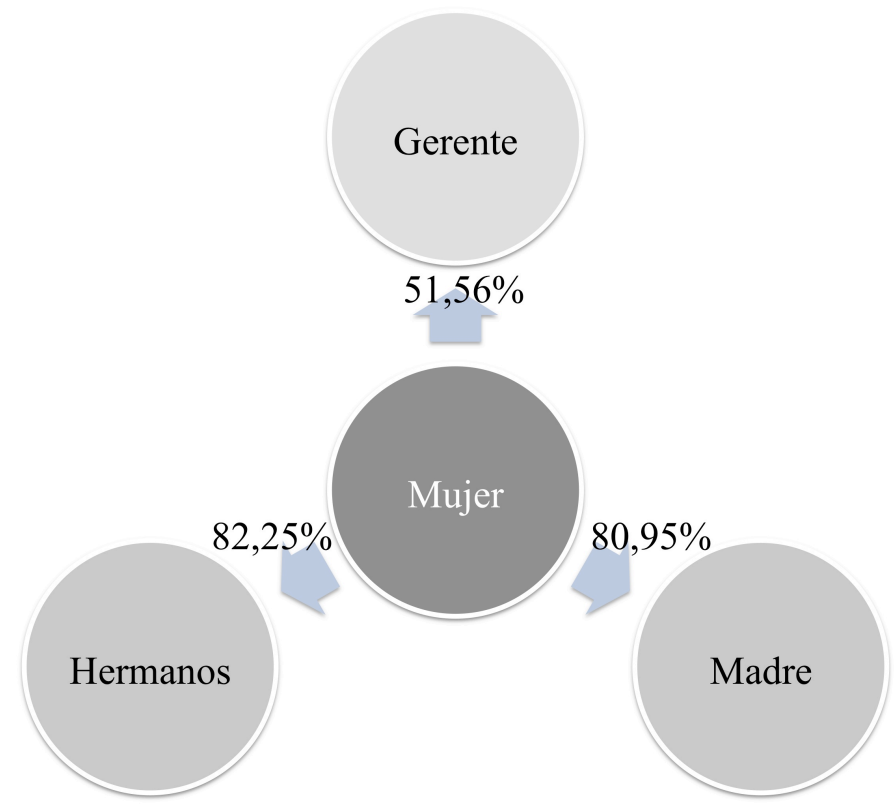

Fuente: elaboración propia
$80,95 \%$ de los casos. Las relaciones con hermanos para las mujeres encuestadas no plantean conflicto en un $85,25 \%$ de los casos. Sin embargo, sí aparecen relaciones conflictivas elevadas con la gerencia en un $48,46 \%$.

Podríamos destacar la percepción de la mujer respecto de su padre de la incapacidad de separar su rol paternal del gerencial cuando se está en la empresa familiar (en un veinte y nueve por ciento de los casos).

\subsection{De la conciliación de la vida familiar}

Pedimos las encuestadas que mostraran su opinión respecto a cuatro afirmaciones planteadas.

-Concilio perfectamente el trabajo en la empresa con el cuidado del hogar y de los hijos (sí $31,93 \%$; no $68,07 \%$ ).

-Trabajo demasiadas horas a la semana, de modo que tengo poco tiempo para atender a mi familia y a la educación de mis hijos (sí $15,13 \%$; no $84,87 \%$ ).

-Decidí reducir la jornada para atender a mis hijos (sí 12,61\%; no 87,39\%).

\section{Fuente: elaboración propia}


-Decidí no asumir más responsabilidades en la empresa para atender a los hijos (sí 10,92\%; no $89,08 \%$ ).

Dado que la maternidad es un factor importante a la hora de contestar a los pronunciamientos expuestos y el tamaño muestral impide realizar cluster o grupos, los resultados resultan únicamente aplicables a la muestra de estudio. Ahora bien, sería interesante abrir la vía de investigación del efecto de la maternidad en la mujer trabajadora en la empresa familiar.

\subsection{De la opinión de la mujer sobre el relevo generacional}

La opinión emitida dada la naturaleza de las preguntas está claramente influenciada por la escala de valores y la dimensión cultural de la muestra. Los resultados, por tanto, deben ser vistos bajo la óptica de la limitación que ésto supone. Fueron realizadas 8 preguntas cuyos resultados tras el pertinente tratamiento de datos mostramos a continuación.

Un 66,33\% afirma que el hecho de ser mujer no le discrimina en la empresa para ocupar puestos de dirección frente a un $27,55 \%$ que se posiciona contrariamente, el $6,12 \%$ no sabe o no contesta.

Un $27,73 \%$ de las mujeres encuestadas opina tener que demostrar más que el hombre en el trabajo para acceder a puestos de dirección frente al $72,27 \%$ que posiciona su respuesta en el lado contrario.

La muestra, atendiendo a las respuestas obtenidas en las dos primeras preguntas no percibe discriminación por razón de sexo en la empresa familiar, en una amplia mayoría de los casos.

Un 57,95\% afirma no estar de acuerdo con que una excesiva protección y una subestimación de sus capacidades le hayan hecho más difícil llegar a dirigir la empresa frente al $25 \%$ que se sitúa en el otro extremo. Un $17,05 \%$ no sabe o no contesta.

El 77,66\% de las mujeres no considera la primogenitura como un factor clave para tomar las riendas del negocio familiar en el proceso sucesorio. Un 15,96\% aún lo considera como factor determinante, y el resto $6,38 \%$ no sabe o no contesta.

Un $94,12 \%$ opina que el hecho de ser mujer no influye en la facilidad de acceder al relevo generacional. Si las mujeres encuestadas no percibían en su amplía mayoría una discriminación negativa respecto del género, aún menos perciben la discriminación positiva, pues el porcentaje obtenido es abrumador $(94,12 \%)$.

El 90,76\% de las mujeres opina que no encontrará oposición o no la encontró en la toma del relevo generacional de la empresa por parte de la figura materna.

En un $14,94 \%$ de los casos las mujeres opinan que nunca dirigirán la empresa familiar por los miedos paternos a dejar el negocio. Un $63,22 \%$ no está de acuerdo con tal afirmación y un $21,84 \%$ no sabe no contesta.

\section{Conclusiones}

La percepción del conflicto por la mujer de la empresa familiar no es elevado. Pese a que se pudiera pensar a priori en la existencia de conflicto con origen en la diferencia de género, hay que recordar que en la relación maternal no se ha planteado conflicto en un $80,95 \%$ de los casos. Las mujeres encuestadas, en una gran mayoría han afirmado no tener conflictos importantes con su madre. Con los hermanos esta cifra se incrementa casi en cinco puntos ya que el dato obtenido para una ausencia de conflicto fraternal es de un $85,25 \%$. Si hay que destacar una elevada percepción de la incapacidad del padre de eliminar su rol paternal en el trabajo.

La mujer decide a entrar a trabajar en la empresa familiar por compromiso, lealtad y valores familiares más que en busca de una flexibilidad y libertad horaria. Los valores familiares se configuran como motor de entrada de la mujer en la empresa familiar frente a la búsqueda de flexibilidad; si bien es cierto, que esta búsqueda de flexibilidad podrá devenir con el tiempo como consecuencia de la formación 
de su propia familia pero no es la causa inicial de entrada a trabajar en la familia empresaria manifestada por las mujeres.

La mujer considera que la formación y la experiencia previa son claves para la entrada a trabajar en la empresa familiar y configuran las principales recomendaciones hacia sus hijas.

La limitación más importante es el tamaño de la muestra efectiva. La población utilizada en el análisis la forman empresas de la provincia de Córdoba que conforman un cuadro de alumnos de siete cursos académicos, aunque tiene la ventaja de ser familiares que trabajan o no en la empresa con el filtro de género necesario para la investigación. A pesar de ser una muestra dirigida pequeña, creemos que la investigación aporta luz en este tipo de análisis y deja la puerta abierta a ulteriores investigaciones.

\section{Bibliografía}

Danes, S.M., Haberman, H. R. \& Mctavish, D. (2005). Gendered discourse about family business. Family Relations, 53, 357-366.

Danes, S.M. \& Mctavish, D. (1997). Role involvement of farm women. Journal of Family and economic Issues, 18(1), 69-84.
Danes , S.M. \& Olson, P.D. (2003). Women's role involvement in family business, family tensions and business success. Family Business Review, 16(1), 53-68.

Kean, R.S., Memeyer \& Maupin, M. (1994). Home based entrepreneurship and multiple roles: an interole conflict. Journal of Consumer Studies and Home Economics, 18, 1-15.

Salganicoff, M. (1990). Women in family business: Challenges and opportunities. Family Business review, 3(2), 197-208.

Stafford, K., Duncan, K.A., Danes, S.M. \& Winter, M. (1999). A research model of sustainable family business. Family Business Review, 12(3), 197-208.

Stoner, C.R., Hartman, R.I. \& Aroca, R. (1990). Work-home conflict in female owners of small business: an exploratory study. Journal of Small Business Management, 28(1), 30-38.

Zuiker, V.S., Lee, Y.G., Olson, P.D., Danes, S.M., Danes, A.N., Vanguilder-Dik \& Katras, M.J. (2002). Business, family and resource intermingling characteristics as predictors of cash flow problems in family owned business. Financial Counseling and Planning, 13(2), 6581.

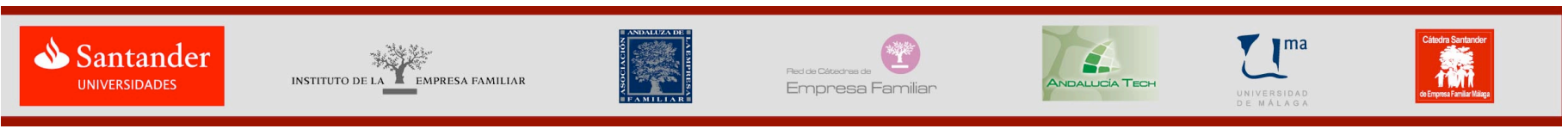

